\title{
Outer stellar disks of lenticular galaxies
}

\author{
Olga K. Sil'chenko \\ Sternberg Astronomical Institute of the Lomonosov Moscow State University, \\ University av. 13, 119991, Moscow, Russia \\ email: olga@sai.msu.su
}

\begin{abstract}
By studying the radial distribution of the properties of stellar populations in 15 nearby S0 galaxies, I have found that the outer stellar disks are mostly old, with SSP-equivalent ages of 8-15 Gyr, often being older than the bulges. This fact throws doubt on the currently accepted paradigm that S0 galaxies were formed at $z=0.4$ through the quenching of star formation in spiral galaxies.
\end{abstract}

Keywords. galaxies: elliptical and lenticular, cD, galaxies: evolution, galaxies: formation

\section{Introduction}

Lenticular galaxies were introduced by Edwin Hubble (1936) as an intermediate type between ellipticals and spirals: they have large-scale stellar disks, as spirals do, but lack blue spiral arms and HII-regions, and they appear smooth and red, as ellipticals do. Nowadays, lenticulars are thought to have been (trans-)formed from spirals through the removal of gas from their disks, and the quenching of star formation. Also, dynamical heating is required to make the S0 disks stable against spiral wave perturbations. There is some evidence that this transformation might have taken place at a redshift of $z \approx 0.4$, within dense environments, groups or clusters, through a sudden shift in the dominance of blue (spiral?) galaxies to a dominance of S0 galaxies (e.g., Fasano et al. 2000, Wilman et al. 2009). But if this scenario is valid, the star formation in the outer disks of nearby S0s should have been going on up to only 4 Gyr ago, and we ought to be able to see intermediate-age stellar populations there now. Using deep long-slit spectroscopy, I have studied the stellar population properties of several nearby S0 galaxies as a function of radial position over several scalelengths of their large-scale disks to check the validity of this paradigm.

\section{Sample}

The sample consists of nearby lenticular galaxies for which deep long-slit spectra were obtained at the Russian $6 \mathrm{~m}$ telescope over the last five years in the context of several observational programs. The main part of the sample are edge-on lenticular galaxies selected for kinematical study by Natalia Sotnikova which have been observed in the context of her observing proposal; I use here these data to derive Lick indices. Four moderately inclined S0 galaxies represent a part of our sample of nearby early-type disk galaxies - group members whose central parts have been studied earlier with the Multi-Pupil Fiber Spectrograph of the $6 \mathrm{~m}$ telescope (Sil'chenko 2006). The galaxies are homogeneously distributed over luminosity - their blue absolute magnitudes being spread from -19 to -21 - and density of environment. We have one galaxy (NGC 4570) in the Virgo cluster where the influence of the intracluster medium is unavoidable, and one 
galaxy (NGC 4111) in the Ursa Major Cluster, where X-ray gas is not detected. Among group galaxies, NGC 524 and NGC 5353 are central group galaxies embedded in Xray haloes; NGC 5308 is a member galaxy of an X-ray bright group, and NGC 502 and IC 1541, though members of rich groups, lie outside their X-ray halo (Osmond \& Ponman 2004, according to our checks of archival ASCA images. NGC 3414 is the central galaxy of a rich group undetected in X-ray, while NGC 2732 is a host of a few faint satellites. NGC 1029, NGC 2549, and NGC 7332 are in triplets. By using the NED environment searcher, we found no galaxies within $300 \mathrm{kpc}$ of NGC 1032 and NGC 1184, so take them to be isolated field galaxies.

\section{Observations}

The long-slit spectral observations were made with the focal reducer SCORPIO (Afanasiev \& Moiseev 2005) installed at the prime focus of the Russian $6 \mathrm{~m}$ telescope (at the Special Astrophysical Observatory of the Russian Academy of Sciences). We exposed a rather narrow spectral range rich in absorpion lines, 4800-5500 $\AA$, which is quite suitable for the study of stellar kinematics and stellar population properties. The slit width was one arcsecond and the spectral resolution about $2 \AA$. The $2 \mathrm{k} \times 2 \mathrm{k}$ and $2 \mathrm{k} \times 4 \mathrm{k}$ CCDs were used as detectors, and the scale along the slit was 0.36 arcsec per pixel. The slit length was about 6 arcminutes so at the edges of the slit we could measure the sky background to subtract from the galaxy spectra. Inhomogeneties in the transparency of the optics and in spectral resolution along the slit were checked with the twilight exposures. The Lick index system was calibrated by observing the standard Lick stars (Worthey et al. 1994) with the same instrumental setup as used for the observations of the galaxies. We calculated the Lick indices H-beta, Mgb, Fe5270, and Fe5335 along the slit up to several exponential scalelengths of the disks. To estimate the radial variations of the SSP-equivalent ages, metallicities and magnesium-to-iron ratios of the stellar populations, I compared the measured Lick indices to the models of old stellar populations by Thomas et al. (2003).

\section{Results and Discussion}

I have measured the Lick indices in 15 S0s along the radius beyond 2-4 exponential scalelengths of their disks, and have estimated the ages and abundances of the stellar populations for the bulges and for the disks. The bulges have solar metallicities or higher, and the disks have solar metallicities or lower. The disk stellar populations demonstrate very high magnesium-to-iron ratios so cannot be descended from prolonged star-formation in spiral galaxies. The SSP-equivalent ages of the disks are mostly old - $60 \%$ of the sample demonstrate ages older than $10 \mathrm{Gyr}$, - and are almost always (except in one case) larger than the SSP-equivalent ages of their corresponding bulges (Fig. 1).

The parameters of the stellar populations of the disks do not show any correlation with the luminosities or masses of the disks. The only correlation found was with the photometric disk scaleheight taken from the decomposition results by Mosenkov et al. (2010). The whole scaleheight range is from $0.3 \mathrm{kpc}$ (found in the disks with the ages of 2-3 Gyr and $[\mathrm{Mg} / \mathrm{Fe}]<+0.2$ ) to $0.6-0.9 \mathrm{kpc}$ (found mostly in the disks with the ages $>10 \mathrm{Gyr}$ and $[\mathrm{Mg} / \mathrm{Fe}]>+0.3)$. All the old disks of our sample galaxies belong to the latter thick disk catagory, while the only two young outer disks, those of NGC 4111 and NGC 7332, are certainly thin disks. The disk of NGC 2732, with its SSP-equivalent age of $8 \mathrm{Gyr}$ and scaleheight of $0.5 \mathrm{kpc}$, is halfway between the thin and thick disks.

Although, many arguments have provided evidence that S0s and spiral galaxies are related, these present results indicate that S0s are progenitors of spirals, opposite to what was thought before. Indeed, if we compare stars of the thick disk of our own Galaxy with 


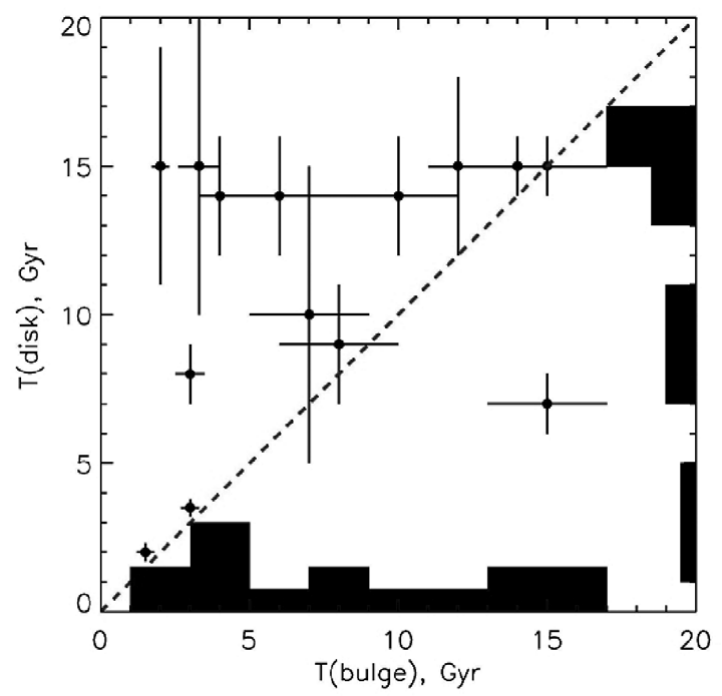

Figure 1. Comparison between the SSP-equivalent ages of the bulges, at $0.5 r_{e}$, and of the large-scale disks for the sample lenticulars. The dashed line is the line of equality; the filled histograms attached to the axes characterize the distributions of the ages of the bulges and disks.

those in the thick outer stellar disks of the S0s studied here we see a full resemblance, with ages of $>10 \mathrm{Gyr},[\mathrm{Mg} / \mathrm{Fe}]>+0.2$, and a total metallicity $[\mathrm{Z} / \mathrm{H}]$ between 0.0 and -0.7 (such parameters of the thick disk of our Galaxy are found by e.g. Bernkopf \& Fuhrmann 2006 or by Schuster et al. 2006). So, if one provides fresh cold gas accretion into the disks of these S0s, after several Gyrs of star formation we would get a typical spiral galaxy, with the thick old stellar disk and thin younger stellar disk. I propose now the following evolutionary sequence: All disk galaxies were S0s immediately after their birth; later, at $z<1$, some of them acquired cold gas accretion sources - these became spirals, - and some of them failed to find such sources - those remained lenticulars. In large clustersize and group-size dark haloes, there is little chance to find external sources of cold gas accretion, due to the hot gas effect, - so in nearby clusters the dominant disk-galaxy population are S0s. It remains an open question as to the nature of these sources of coldgas prolonged accretion. They may be filaments formed in the cosmological development of structure (Dekel \& Birnboim 2006) or rich systems of irregular-type dwarf satellites which have been merging with the host one after another.

\section{References}

Afanasiev, V. L. \& Moiseev A. V. 2005, Astron. Lett., 31, 194

Bernkopf, J. \& Fuhrmann K. 2006, MNRAS, 369, 673

Dekel, A. \& Birnboim, Yu. 2006, MNRAS, 368, 2

Fasano, G., Poggianti, B. M., Couch, W. J., et al. 2000, ApJ, 542, 673

Hubble, E. 1936, The Realm of the Nebulae (New Haven: Yale Univ. Press)

Mosenkov, A. V., Sotnikova, N. Ya., \& Reshetnikov, V. P. 2010, MNRAS, 401, 559

Osmond, J. P. F. \& Ponman T. J. 2004, MNRAS, 350, 1511

Schuster, W. J., Moitinho, A., Marquez, A., Parrao, L., \& Covarrubias, E. 2006, A\&A A, 445, 939

Sil'chenko, O. K. 2006, ApJ, 641, 229

Thomas, D., Maraston, C., \& Bender, R. 2003, MNRAS, 339, 897

Wilman, D. J., Oemler, A., Mulchaey, J. S., et al. 2009, ApJ, 692, 298

Worthey, G., Faber, S. M., González, J. J., \& Burstein, D. 1994, ApJS, 94, 687 